\title{
Thickness of Subcutaneous Fat Is a Predictive Factor of Incisional Surgical Site Infection in Crohn's Disease Surgery: A Retrospective Study
}

\author{
Xingchen Cai, ${ }^{1}$ Weisong Shen, ${ }^{2}$ Zhen Guo, ${ }^{2}$ Yi Li, ${ }^{2}$ Lei Cao, ${ }^{2}$ Jianfeng Gong, \\ and Weiming Zhu $\mathbb{1}^{2}$ \\ ${ }^{1}$ Department of General Surgery, Jinling Hospital, Nanjing Medical University, Nanjing, China \\ ${ }^{2}$ Department of General Surgery, Jinling Hospital, Medical School of Nanjing University, Nanjing, China \\ Correspondence should be addressed to Weiming Zhu; juwiming@nju.edu.cn
}

Received 1 February 2018; Revised 29 April 2018; Accepted 30 May 2018; Published 22 July 2018

Academic Editor: Cristiano Pagnini

Copyright (C) 2018 Xingchen Cai et al. This is an open access article distributed under the Creative Commons Attribution License, which permits unrestricted use, distribution, and reproduction in any medium, provided the original work is properly cited.

\begin{abstract}
Background. Incisional surgical site infection (iSSI) is a frequent postoperative complication of abdominal surgeries in patients with Crohn's disease (CD). In this study, we investigated the association between thickness of subcutaneous fat (TSF) and iSSI in patients with CD undergoing intestinal resections. Patients and Methods. Patients with CD who had undergone abdominal surgery from January 2014 to January 2017 were included in this retrospective study. Patients' TSF and other possible predictors of iSSI, including clinical characteristics, preoperative medications, hematological index, surgery-related data, and postoperative outcomes, were collected. Univariate and multivariate statistical analyses were used to examine the potential factors. Receiver operating characteristic (ROC) curve analysis was used to evaluate the predictive value of factors. Results. The patient cohort comprised 246 patients (167 male (67.9\%); mean age 35.7 \pm 12.4 years; mean disease duration $69.6 \pm 60.8$ months). The incidence of iSSI was $24.8 \%(61 / 246)$. TSF was a significant predictor of iSSI (OR 1.079, 95\% CIs $(1.020,1.142), P=0.008)$, being $13.7 \mathrm{~mm}$ in patients with iSSI and $9.9 \mathrm{~mm}$ in those without iSSI $(P<0.001)$. Additionally, C-reactive protein $(\mathrm{CRP})$ concentrations (OR 1.059, $P=0.003$ ) were also possible predictors of iSSI, as indicated by both univariate and multivariate analysis. A model of iSSI comprising TSF and CRP concentrations was moderately accurate (AUC 0.827, CIs (0.766, 0.888)). Conclusions. Preoperative TSF and CRP independently affect iSSI in patients with CD undergoing intestinal resections.
\end{abstract}

\section{Introduction}

Crohn's disease (CD), a chronic inflammatory bowel disorder, involves the whole digestive tract. In recent years, there has been ongoing development of medical treatments, particularly immunosuppressant and biological agents $[1,2]$. However, nearly $60 \%$ of patients with $\mathrm{CD}$ have to undergo at least one abdominal operation [3].

Surgical site infection (SSI) is the commonest infectious complication after abdominal surgery for colorectal cancer and diverticulitis, the incidence being 2\%-5\% [4]. Furthermore, it is responsible for the highest proportion of costs of hospital-acquired infections [4]. At 14\%-24\%, its incidence is much higher in patients with $\mathrm{CD}$ who have undergone abdominal surgery than in other patients [5-7]. Incisional
SSI (iSSI) is a significant form of SSI. Therefore, identifying risk factors for iSSI could help to prevent this complication of surgery in patients with $\mathrm{CD}$.

A range of risk factors contributes to iSSI, including preoperative malnutrition, obesity, intra-abdominal abscess, preoperative blood glucose concentration, and intraoperative blood transfusions [8]. Although patients with $\mathrm{CD}$ tend to have multiple risk factors for iSSI, such as malnutrition and immunosuppressive therapies, there have been few studies of risk factors in patients with CD undergoing surgery.

The thickness of subcutaneous fat (TSF) is a common index of nutritional status [9], and previous studies have reported that TSF is a strong risk factor for iSSI after open gastrectomy for gastric cancer and elective colorectal surgery for colorectal cancer $[10,11]$. Because of the characteristics of 
their disease, TSF differs between patients with CD and those with other diseases. To our knowledge, no studies have investigated the relationship between TSF and rate of the iSSI in patients with CD.

The aim of the current study was therefore to focus on investigating any correlation between TSF and the iSSI in $\mathrm{CD}$ patients together with assessing other potential risk factors for iSSI, such as smoking, blood loss, and preoperative albumin and C-reactive protein (CRP) concentrations.

\section{Materials and Methods}

2.1. Patients. Data on patients with CD who had undergone intestinal resections from January 2014 to January 2017 in the Inflammatory Bowel Disease Center of Jinling Hospital were retrospectively collected. All study patients had undergone routine complete abdominal CT or MRI scans within the two weeks before surgery. This study was approved by the Jinling Hospital Ethics Committee.

Patients who were to undergo elective surgery received optimal therapy aimed at improving their nutritional status and reducing systematic inflammation for at least 3 weeks prior to surgery. The strategies included nutritional therapy, drainage of abscesses, weaning off steroids for $>4$ weeks in patients who had been taking $>20 \mathrm{mg}$ prednisolone for $>6$ weeks, withdrawal of antitumor necrosis factor (TNF) for $>3$ months, antibiotics, and smoking cessation. Indications for emergency surgery included acute intestinal perforation and massive hemorrhage and prevented implementation of the full range of strategies listed above.

Preoperative nutritional support was provided as enteral nutrition (EN) (Nutrison Fibre, Enteral Nutrition Suspension; Nutricia Company, Amsterdam, the Netherlands) through a nasogastric tube in all patients who could tolerate it. Some patients initially received total parenteral nutrition (TPN) and then gradually transited to EN (Peptisorb Liquid, Enteral Nutrition Suspension; Nutricia Company, Amsterdam, the Netherlands). Patients with abdominal abscesses underwent preoperative percutaneous drainage if possible and antibiotic therapy. All operations were performed by the same surgical group, and perioperative management was in accordance with the principle of enhanced recovery after surgery (ERAS). All patients except for those undergoing emergency surgery were fed glucose and sodium chloride solution with nasogastric tube for 24 hours prior to surgery rather than undergoing preoperative mechanical bowel preparation. Incision protection sleeves were used in laparotomies. Prophylactic antibiotics (a single dose of secondgeneration cephalosporins) were typically administered 30 minutes preoperatively and by intravenous drip on the first and second postoperative days.

2.2. Definitions. Diagnoses of iSSI were made in accordance with the guidelines of the Centers for Disease Control and Prevention, Public Health Service, US Department of Health and Human Services [12]. Incisional SSIs were further subclassified as superficial or deep incisional SSI. Superficial incisional SSI was defined as occurring within 30 days of surgery, infection involving only skin or subcutaneous tissue of an incision, and at least one of the following: (1) purulent drainage; (2) organisms isolated from an aseptically obtained culture of fluid or tissue from the superficial part of an incision; or (3) signs or symptoms of infection, including pain, tenderness, localized swelling, redness, heat, and superficial part of an incision deliberately opened by the surgeon, unless the incision was culture-negative. Deep incisional SSI was defined as infection involving the fascia of the incision and at least one of the following: (1) purulent drainage deep in an incision; (2) spontaneous dehiscence or deliberate opening of a deep incision by a surgeon in a patient with the following signs or symptoms: fever $\left(>38^{\circ} \mathrm{C}\right)$ and localized pain or tenderness, unless the site is culture-negative; (3) an abscess or other evidence of infection involving a deep incision is found on direct examination, during reoperation, or by histopathologic or radiologic examination. Diagnoses of an iSSI were made by a surgeon or attending physician.

The area selected for measurement of TSF by preoperative CT or MRI scans was one centimeter to the right of the umbilical plane, and the thickness was defined as the distance between the posterior lines of the dermis and the superficial fascia of musculus rectus abdominis [13]. Measurements were performed by two authors (Cai XC and Shen WS) using the Adobe Photoshop (version 16.0.0.88).

2.3. Data Collection. Specific variables recorded for each patient were as follows: age, gender, duration of disease, disease behavior, disease location, perianal disease, intraabdominal septic complications (IASCs) including anastomotic leak, intra-abdominal abscess, or enterocutaneous fistula, previous abdominal surgery, BMI, smoking habits, preoperative hospitalizations, preoperative nutritional support and drug administration, surgical approach, length and class of incision, duration of operation, intraoperative blood loss, anastomosis position, postoperative IASCs, and stoma creation. Preoperative leukocyte counts and CRP, serum albumin, and blood glucose were all assessed within the 3 days before surgery.

2.4. Statistical Analysis. All data were analyzed using the SPSS version 22.0 (SPSS, Chicago, IL, USA). Continuous parametric variables are presented as the mean $\pm S D$, whereas nonparametric data are presented as 5\% to 95\% confidence intervals. The series mean was chosen to replace missing values. Unpaired $t$-tests were used to compare parametric variables and the Mann-Whitney $U$ test for nonparametric data. Categorical data were analyzed by the $\chi^{2}$ tests and contingency tables. Univariate and multivariate logistic regression analyses were performed to identify possible predictors of postoperative iSSI. Backward Wald was selected to choose confounders, and only variables with $P<0.10$ in univariate analysis were assessed by multivariate logistic regression analysis. Predictive accuracy was assessed by receiver operating characteristic (ROC) curve analysis. Multi-index combined ROC curve analysis was first performed with multivariate logistic regression analysis and output probabilities of predicted values, after which, probabilities were further assessed by constructing ROC curves. Overall, $P<0.05$ was considered to denote statistical significance. 


\section{Results}

3.1. Clinical Characteristics of Patients. The study cohort comprised 246 patients (167 men, 67.9\%) of average age 35.7 years (8 to 71 years). The disease duration was $69.6 \pm$ 60.8 months and the average TSF $10.87 \mathrm{~mm}$ (range $0.76-$ $37.92 \mathrm{~mm}$ ). The incidence of iSSI in this cohort was $24.8 \%$ (61/246).

Relevant patient data, preoperative medications, and operative data are summarized in Table 1 and hematological indexes in Table 2. TSF was $13.7 \mathrm{~mm}$ in patients who developed an iSSI and $9.9 \mathrm{~mm}$ in those who did not $(P<0.001)$. Univariate analysis showed that the potential risk factors for iSSI $(P<0.1)$ in patients with CD included previous abdominal surgery, length of incision, type of incision, preoperative CRP, hemoglobin, and blood glucose. To investigate associations between individual risk factors and development of iSSI, the factors were subjected to multivariate analysis, as shown in Table 3 . TSF was a significant predictor of iSSI (OR 1.079, 95\% CIs (1.020, 1.142), $P=$ 0.008 ). Additionally, preoperative CRP concentrations were much higher in the iSSI group (OR 1.059, CIs (1.019, 1.100), $P=0.003)$.

3.2. ROC Curve Analysis. ROC curves were constructed to determine the predictive value of TSF for iSSI in patients with $\mathrm{CD}$ (Figure 1). The area under the curve (AUC) was 0.646 (CIs $(0.583,0.706))$, the sensitivity of the cut-off value of $10.2 \mathrm{~mm}$ being $59.1 \%$ and the specificity $63.8 \%$. Furthermore, the negative predictive value (NPV) was $82.5 \%$ and the positive predictive value (PPV) only $35.0 \%$. An attempt was made to build a model for iSSI prediction comprising preoperative TSF and CRP concentrations. ROC curves were then constructed to analyze the predictive value, as shown in Figure 2. In this model, the cut-off point was 0.152 and AUC 0.827 (CIs $(0.766,0.888)$ ). Cut-off point 0.152 was corresponding to TSF cut-off value $10.2 \mathrm{~mm}$ and CRP cut-off value $8.6 \mathrm{mg} / \mathrm{L}$. The sensitivity was $90.2 \%$ and the specificity $63.2 \%$. The NPV of the cut-off point was $95.1 \%$ and the PPV 44.7\%, indicating that the model has a higher predictive value for iSSI than TSF or CRP alone.

3.3. Predictors of iSSI. Preoperative TSF and CRP concentrations were classified according to cut-off values of $10.2 \mathrm{~mm}$ and $8.6 \mathrm{mg} / \mathrm{L}$, respectively. Multivariate analysis was performed to identify predictors significantly associated with iSSI; these are presented in Table 4. TSF (OR 2.519, CIs (1.350, 4.698), $P=0.004)$ and CRP (OR 4.556, CIs (2.378, $8.728), P<0.001)$ were both associated with an increased risk of iSSI.

\section{Discussion}

Surgery for $\mathrm{CD}$ is challenging, often being accompanied by multiple postoperative complications. Infective complications are an important type of postoperative complication in these patients, previous studies having mainly focused on exploring risk factors for development of intra-abdominal sepsis [14-16]. Postoperative iSSI, the major form of infective complication, increases hospitalization costs, prolongs hospital stay, and negatively affects patients' quality of life. The incidence of iSSI is reportedly much higher in patients with $\mathrm{CD}$ than in those with other diseases [5, 17]; however, there are few studies on iSSI after abdominal surgery for $\mathrm{CD}$. In the present retrospective study of patient data, our primary focus was on the relationship between postoperative iSSI and thickness of abdominal subcutaneous fat in patients with $\mathrm{CD}$. First, the incidence of iSSI in our patients with $\mathrm{CD}$ who had undergone intestinal resection was $24.8 \%$ (61/246), this rate being quite close to that reported by Bhakta et al. [5]. Second, we found preoperative TSF to be an independent risk factor for iSSI after surgery for CD. Third, preoperative CRP concentrations were also predictors of iSSI, and a model including TSF and CRP concentrations had better predictive value (AUC 0.827) than these predictors alone.

The body fat distribution of patients with $\mathrm{CD}$, especially those with aggressive disease, differs from that of other persons, this being mainly characterized by a higher ratio of visceral to subcutaneous fat [18]. TSF is associated with BMI, and several studies have shown that TSF is an independent risk factor for iSSI after elective colorectal surgeries $[11,19]$. A relationship between iSSI and TSF makes intuitive sense and has long been proven. Soper and Bump [20] reported that only patients with depth of subcutaneous fat $>3 \mathrm{~cm}$ developed iSSI after undergoing abdominal hysterectomy. Fujii et al. [11] reported a cut-off value for TSF of $20 \mathrm{~mm}$ in Asian patients who developed iSSI after undergoing elective colorectal surgery. However, $\mathrm{CD}$ is a type of wasting disease, affecting individuals often having very thin abdominal subcutaneous fat, even as thin as a wafer. In our 246 patients, the minimum TSF was only $0.76 \mathrm{~mm}$ and the mean $10.87 \mathrm{~mm}$. Therefore, previously demonstrated associations between TSF and iSSI cannot validly be assumed to apply to patients with CD. We found that TSF is an independent risk factor for iSSI in patients undergoing surgery for $\mathrm{CD}$, the cut-off point being $10.2 \mathrm{~mm}$. This cut-off value is markedly lower than the $20 \mathrm{~mm}$ reported by Fujii et al., indicating that CD differs from other diseases such that it is inappropriate to mechanically predict postoperative complications in patients using predictive models developed for other diseases.

CRP is synthesized in hepatocytes, and serum concentrations increase rapidly during inflammatory responses [21]; thus, serum CRP concentration often plays a role as a marker of inflammation in a clinical context. Given that peak CRP concentrations occur about 2 days after the initiation of an acute inflammatory response and facilitate detection of such responses, CRP concentrations can be used to predict postoperative infective complications [22]. Serum CRP concentration on the fourth postoperative day can be used to eliminate postoperative infectious complications [23]. Additionally, CRP concentration is reportedly the most frequently used noninvasive biomarker for evaluating the activity of $\mathrm{CD}$. Furthermore, in a previous study, we found that preoperative CRP concentration is a practical predictor of intraabdominal septic complications after performing primary anastomoses in patients with CD [14]. In the present study, we found that preoperative CRP concentration is a predictor of postoperative iSSI in patients with CD. This finding is in 
TABLE 1: Clinical and operational characteristics of patients in the research group.

\begin{tabular}{|c|c|c|c|}
\hline \multirow{2}{*}{ Variable } & \multicolumn{2}{|c|}{ Incisional SSI } & \multirow{2}{*}{$P$ value } \\
\hline & Present $(n=61)$ & Absent $(n=185)$ & \\
\hline Gender (male/female) & $41 / 20$ & $126 / 59$ & 0.507 \\
\hline Age (years) & $36.5 \pm 13.3$ & $35.5 \pm 12.7$ & 0.593 \\
\hline Duration of disease (months) & $65.6 \pm 60.5$ & $70.9 \pm 61.1$ & 0.554 \\
\hline Thickness of subcutaneous fat (mm) & $13.7 \pm 7.7$ & $9.9 \pm 6.1$ & $<0.001$ \\
\hline Disease behavior, $n(\%)$ & & & 0.203 \\
\hline Stricture & $38(62.3)$ & $87(47.0)$ & - \\
\hline Penetration & $21(34.4)$ & $91(49.2)$ & - \\
\hline Others & $2(3.3)$ & $7(3.8)$ & - \\
\hline Disease location, $n(\%)$ & & & 0.373 \\
\hline Ileum & $17(27.9)$ & $65(35.1)$ & - \\
\hline Colon & $6(9.8)$ & $25(13.5)$ & - \\
\hline Ileocolon & $38(62.3)$ & $93(50.3)$ & - \\
\hline Upper digestive tract & $0(0)$ & $2(1.1)$ & - \\
\hline Perianal disease, $n(\%)$ & $15(24.6)$ & $61(33.0)$ & 0.219 \\
\hline Abdominal abscess, $n$ (\%) & $10(16.4)$ & $15(8.1)$ & 0.063 \\
\hline Previous abdominal surgery for $\mathrm{CD}, n(\%)$ & & & 0.119 \\
\hline 0 & $25(41.0)$ & $102(55.1)$ & - \\
\hline 1 & $26(42.7)$ & $65(35.2)$ & - \\
\hline$>1$ & $10(16.3)$ & $18(9.7)$ & - \\
\hline Smoking, $n(\%)$ & $10(16.3)$ & $15(8.1)$ & 0.063 \\
\hline Length of preoperative hospitalization (days) & $9.7 \pm 16.5$ & $8.1 \pm 4.6$ & 0.222 \\
\hline BMI $\left(\mathrm{kg} / \mathrm{m}^{2}\right)$ & $17.4 \pm 5.5$ & $16.7 \pm 5.0$ & 0.380 \\
\hline Preoperative PN, $n(\%)$ & $13(21.3)$ & $34(18.4)$ & 0.613 \\
\hline Preoperative EN, $n(\%)$ & $47(77.0)$ & $157(84.9)$ & 0.159 \\
\hline \multicolumn{4}{|l|}{ Preoperative drug administration, $n(\%)$} \\
\hline 5 -ASA & $15(24.6)$ & $59(31.9)$ & 0.281 \\
\hline Steroids & $5(8.2)$ & $12(6.5)$ & 0.771 \\
\hline Immunosuppressant & $20(32.8)$ & $60(32.4)$ & 0.959 \\
\hline Anti-TNF $\alpha$ antibody & $6(9.8)$ & $12(6.5)$ & 0.400 \\
\hline Emergency operation, $n(\%)$ & $5(8.2)$ & $11(5.9)$ & 0.553 \\
\hline Surgical approach, $n(\%)$ & & & 0.117 \\
\hline Laparotomy & $52(85.2)$ & $140(75.7)$ & - \\
\hline Laparoscopic & $9(14.8)$ & $45(24.3)$ & - \\
\hline Anastomosis position, $n(\%)$ & & & 0.255 \\
\hline Nonanastomosis & $11(18.0)$ & $39(21.1)$ & - \\
\hline Ileal anastomosis & $15(24.6)$ & $62(33.5)$ & - \\
\hline Ileocolostomy & $35(57.4)$ & $84(45.4)$ & - \\
\hline Stoma creation, $n(\%)$ & & & 0.532 \\
\hline Ileostomy & $13(21.3)$ & $44(23.8)$ & - \\
\hline Colostomy & $4(6.6)$ & $3(1.7)$ & - \\
\hline Length of incision $(\mathrm{cm})$ & $12.2 \pm 3.5$ & $10.6 \pm 4.2$ & 0.009 \\
\hline Incision class, $n(\%)$ & & & 0.011 \\
\hline Contaminated & $51(83.6)$ & $174(94.1)$ & - \\
\hline Dirty & $10(16.4)$ & $11(5.9)$ & - \\
\hline Duration of operation (min) & $148.6 \pm 49.5$ & $141.2 \pm 52.5$ & 0.337 \\
\hline Intraoperative blood loss (mL) & $218.2 \pm 157.4$ & $182.5 \pm 116.2$ & 0.059 \\
\hline Postoperative transferred to ICU, $n(\%)$ & $10(16.4)$ & $19(10.3)$ & 0.189 \\
\hline Postoperative IASCs, $n(\%)$ & & & 0.020 \\
\hline
\end{tabular}


TABLE 1: Continued.

\begin{tabular}{lccc}
\hline Variable & Incisional SSI & & $P$ value \\
\hline Anastomotic leak & Present $(n=61)$ & Absent $(n=185)$ & $2(1.1)$ \\
Intra-abdominal abscess & $3(8.2)$ & $1(0.5)$ & - \\
Enterocutaneous fistula & $3(4.9)$ & $1(0.5)$ & - \\
Reoperation, $n(\%)$ & $1(1.6)$ & $1(0.5)$ & 0.153 \\
\hline
\end{tabular}

Data are reported as the number of patients (\%) or mean \pm SD. SSI: surgical site infection; CD: Crohn's disease; BMI: body mass index; PN: parenteral nutrition; EN: enteral nutrition; IASCs: intra-abdominal septic complications.

TABLE 2: Hematological index.

\begin{tabular}{lccc}
\hline Variable & Present $(n=61)$ & Incisional SSI & Absent $(n=185)$ \\
\hline Preoperative leukocyte $\left(10^{9} / \mathrm{L}\right)$ & $6.1 \pm 3.9$ & $5.4 \pm 2.5$ & 0.154 \\
Preoperative CRP $(\mathrm{mg} / \mathrm{L})$ & $32.0 \pm 44.5$ & $6.7 \pm 18.9$ & $<0.001$ \\
Preoperative albumin $(\mathrm{g} / \mathrm{L})$ & $36.7 \pm 7.5$ & $37.0 \pm 5.7$ & 0.723 \\
Preoperative hemoglobin $(\mathrm{g} / \mathrm{L})$ & $112.0 \pm 20.2$ & $118.3 \pm 16.3$ & 0.014 \\
Preoperative blood glucose $(\mathrm{mmol} / \mathrm{L})$ & $5.8 \pm 1.7$ & $5.2 \pm 1.2$ & 0.029 \\
\hline
\end{tabular}

Data are reported as mean \pm SD. SSI: surgical site infection; CRP: C-reactive protein.

TABLE 3: Multivariable logistic regression analysis of predictors of iSSI in patients with CD after abdominal surgery.

\begin{tabular}{lccc}
\hline Variable & $95 \%$ CI & OR & $P$ value \\
\hline Thickness of subcutaneous fat & $1.020-1.142$ & 1.079 & 0.008 \\
Abdominal abscess & $0.236-4.500$ & 1.030 & 0.969 \\
Smoking & $0.564-5.116$ & 1.698 & 0.347 \\
Length of incision & $0.938-1.153$ & 1.040 & 0.458 \\
Incision class & $0.113-6.450$ & 0.854 & 0.878 \\
Blood loss & $0.998-1.004$ & 1.001 & 0.497 \\
Preoperative CRP & $1.019-1.100$ & 1.059 & 0.003 \\
Preoperative hemoglobin & $0.975-1.027$ & 1.001 & 0.953 \\
Preoperative blood glucose & $0.924-1.597$ & 1.215 & 0.164 \\
\hline
\end{tabular}

OR: odds ratio; CI: confidence interval; $\mathrm{CD}$ : Crohn's disease; CRP: C-reactive protein; IASCs: intra-abdominal septic complications.

line with our hypothesis that preoperative CRP concentration reflects the activity and severity of $\mathrm{CD}$.

Whether preoperative administration of infliximab influences the incidence of postoperative iSSI remains controversial. Some meta-analyses have indicated that preoperative infliximab is associated with increased risk of infectious complications in patients with $\mathrm{CD}$ who undergo surgeries $[24,25]$. However, the findings of another meta-analysis suggested that infliximab does not increase the risks of postoperative infective complications [26]. Uchino et al. reported that preoperative administration of infliximab is not a risk factor for iSSI [27]. Thus, the relationship between such medications and postoperative iSSI is still under discussion. In our study, infliximab administration showed no correlation with postoperative iSSI in patients with CD.

The incidence of postoperative IASCs in this cohort was $4.5 \%(11 / 246)$, which is comparable to the $5 \%-20 \%$ reported by previous studies $[15,16,28]$. Only three patients with

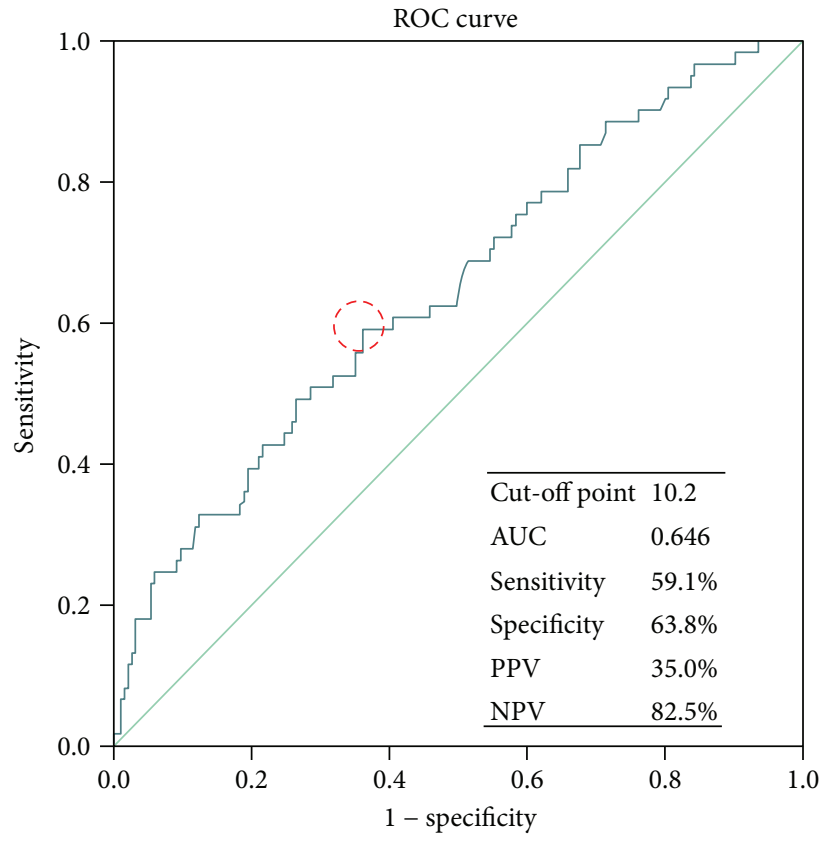

FIgURE 1: ROC curve showing preoperative TSF predictive value of postoperative iSSI. TSF: thickness of subcutaneous fat; ROC: receiver operating characteristic; AUC: area under the curve; PPV: positive predictive value; NPV: negative predictive value.

IASCs required revision surgery, the remaining IASCs resolved with percutaneous drainage. Though postoperative IASCs were strongly associated with iSSI, especially deep iSSI, we considered that IASCs have limited predictive value because they develop later than other indexes. We therefore did not include postoperative IASCs as a predictor of iSSI.

Our study had some limitations. First, it was a singlecenter retrospective study. However, it was relatively large, 


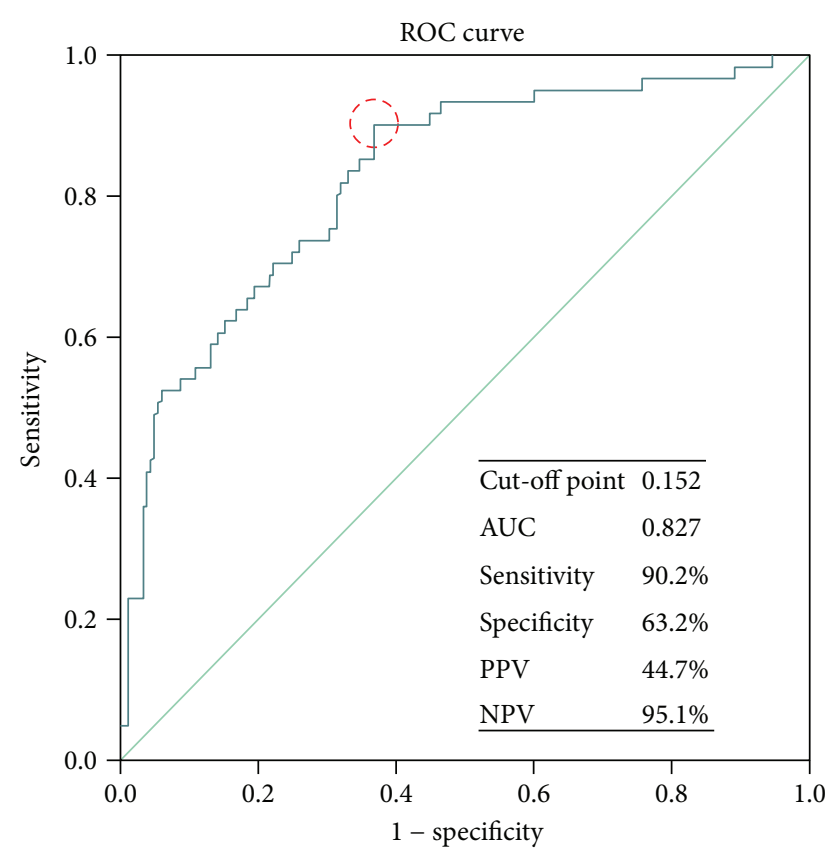

FIGURE 2: ROC curve showing the model within preoperative TSF and CRP levels predictive value of postoperative iSSI. CRP: Creactive protein; ROC: receiver operating characteristic; AUC: area under the curve; PPV: positive predictive value; NPV: negative predictive value.

TABLE 4: Multivariable logistic regression analysis of iSSI predictors classified according to cut-off value.

\begin{tabular}{lccc}
\hline Variable & $95 \%$ CI & OR & $P$ value \\
\hline Thickness of subcutaneous fat & $1.350-4.698$ & 2.519 & 0.004 \\
Preoperative CRP & $2.378-8.728$ & 4.556 & $<0.001$ \\
\hline
\end{tabular}

OR: odds ratio; CI: confidence interval: CRP: C-reactive protein.

including 246 patients. However, our findings are less persuasive than those of a prospective study would be and lack widespread applicability. Second, we found that TSF is a predictor of iSSI; we did not evaluate associations between iSSI severity and amount of TSF. Third, only 16 patients in our series had undergone emergency abdominal operation surgery, which may have influenced the accuracy of our data and the universality of our results. Despite these limitations, our findings indicate that TSF has predictive value for postoperative iSSI in patients with CD.

\section{Conclusion}

This study is the first to investigate the relationship between TSF and iSSI after abdominal surgery for CD. Our results suggest that preoperative TSF and CRP concentrations can predict development of postoperative iSSI. We therefore recommend the use of incision protection supplementary materials, more frequent checking of incisions, and prompt treatment to minimize the incidence of postoperative iSSI in patients with Crohn's disease, preoperative TSF $>10.2 \mathrm{~mm}$, and $\mathrm{CRP}>8.6 \mathrm{mg} / \mathrm{L}$.

\section{Data Availability}

The data used to support the findings of this study are available from the corresponding author upon request.

\section{Disclosure}

The authors disclose that there is no financial and personal relationships with other people or organizations that could potentially and inappropriately influence (bias) their work and conclusions.

\section{Conflicts of Interest}

The authors declare that they have no conflicts of interest.

\section{Authors' Contributions}

Xingchen Cai, Weisong Shen, and Zhen Guo conceived and designed the study. Xingchen Cai collected the data and wrote the manuscript. Weisong Shen analyzed and interpreted the data. Yi Li, Lei Cao, and Jianfeng Gong provided critical revisions that were important for the intellectual content. Weiming Zhu approved the final version of the manuscript. Xingchen Cai and Weisong Shen contributed equally to this work.

\section{Acknowledgments}

The authors thank Dr. Trish Reynolds, MBBS, FRACP, from Liwen Bianji, Edanz Group China (http://www.liwenbianji. $\mathrm{cn} / \mathrm{ac}$ ), for editing the English text of a draft of this manuscript. This study was funded by the National Natural Science Foundation of China (81500429 and 81570500).

\section{References}

[1] D. C. Baumgart, "Crohn's disease," The Lancet, vol. 380, no. 9853, pp. 1590-1605, 2012.

[2] T. Dassopoulos, S. Sultan, Y. T. Falck-Ytter, J. M. Inadomi, and S. B. Hanauer, "American Gastroenterological Association Institute technical review on the use of thiopurines, methotrexate, and anti-TNF- $\alpha$ biologic drugs for the induction and maintenance of remission in inflammatory Crohn's disease," Gastroenterology, vol. 145, no. 6, pp. 1464-1478.e5, 2013.

[3] L. Peyrin-Biroulet, W. S. Harmsen, W. J. Tremaine, A. R. Zinsmeister, W. J. Sandborn, and E. V. Loftus, "Surgery in a population-based cohort of Crohn's disease from Olmsted County, Minnesota (1970-2004)," The American Journal of Gastroenterology, vol. 107, no. 11, pp. 1693-1701, 2012.

[4] K. A. Ban, J. P. Minei, C. Laronga et al., "American College of Surgeons and Surgical Infection Society: surgical site infection guidelines, 2016 update," Journal of the American College of Surgeons, vol. 224, no. 1, pp. 59-74, 2017.

[5] A. Bhakta, M. Tafen, O. Glotzer et al., "Increased incidence of surgical site infection in IBD patients," Diseases of the Colon \& Rectum, vol. 59, no. 4, pp. 316-322, 2016.

[6] M. Uchino, H. Ikeuchi, T. Tsuchida, K. Nakajima, N. Tomita, and Y. Takesue, "Surgical site infection following surgery for inflammatory bowel disease in patients with clean- 
contaminated wounds," World Journal of Surgery, vol. 33, no. 5, pp. 1042-1048, 2009.

[7] T. Serradori, A. Germain, M. L. Scherrer et al., "The effect of immune therapy on surgical site infection following Crohn's disease resection," British Journal of Surgery, vol. 100, no. 8, pp. 1089-1093, 2013.

[8] L. Neumayer, P. Hosokawa, K. Itani, M. el-Tamer, W. G. Henderson, and S. F. Khuri, "Multivariable predictors of postoperative surgical site infection after general and vascular surgery: results from the patient safety in surgery study," Journal of the American College of Surgeons, vol. 204, no. 6, pp. 1178-1187, 2007.

[9] D. Q. Holt, B. J. Strauss, and K. K. Lau, "Body composition analysis using abdominal scans from routine clinical care in patients with Crohn's disease," Scandinavian Journal of Gastroenterology, vol. 51, no. 7, pp. 842-847, 2016.

[10] M. Tokunaga, N. Hiki, T. Fukunaga, T. Ogura, S. Miyata, and T. Yamaguchi, "Effect of individual fat areas on early surgical outcomes after open gastrectomy for gastric cancer," British Journal of Surgery, vol. 96, no. 5, pp. 496-500, 2009.

[11] T. Fujii, S. Tsutsumi, A. Matsumoto et al., "Thickness of subcutaneous fat as a strong risk factor for wound infections in elective colorectal surgery: impact of prediction using preoperative CT," Digestive Surgery, vol. 27, no. 4, pp. 331-335, 2010.

[12] A. J. Mangram, T. C. Horan, M. L. Pearson, L. C. Silver, and W. R. Jarvis, "Guideline for prevention of surgical site infection, 1999," American Journal of Infection Control, vol. 27, no. 2, pp. 97-134, 1999.

[13] A. M. Gradmark, A. Rydh, F. Renström et al., "Computed tomography-based validation of abdominal adiposity measurements from ultrasonography, dual-energy X-ray absorptiometry and anthropometry," British Journal of Nutrition, vol. 104, no. 4, pp. 582-588, 2010.

[14] L. Zuo, Y. Li, H. Wang et al., "A practical predictive index for intra-abdominal septic complications after primary anastomosis for Crohn's disease: change in C-reactive protein level before surgery," Diseases of the Colon \& Rectum, vol. 58, no. 8, pp. 775-781, 2015.

[15] I. Iesalnieks, A. Kilger, H. Glaß et al., "Intraabdominal septic complications following bowel resection for Crohn's disease: detrimental influence on long-term outcome," International Journal of Colorectal Disease, vol. 23, no. 12, pp. 1167-1174, 2008.

[16] P. Myrelid, G. Olaison, R. Sjödahl, P. O. Nyström, S. Almer, and P. Andersson, "Thiopurine therapy is associated with postoperative intra-abdominal septic complications in abdominal surgery for Crohn's disease," Diseases of the Colon \& Rectum, vol. 52, no. 8, pp. 1387-1394, 2009.

[17] C. Lau, M. Dubinsky, G. Melmed et al., "The impact of preoperative serum anti-TNF $\alpha$ therapy levels on early postoperative outcomes in inflammatory bowel disease surgery," Annals of Surgery, vol. 261, no. 3, pp. 487-496, 2015.

[18] B. Erhayiem, R. Dhingsa, and C. J. Hawkey, "Ratio of visceral to subcutaneous fat area is a biomarker of complicated Crohn's disease," Clinical Gastroenterology and Hepatology, vol. 9, no. 8, pp. 684-687.e1, 2011.

[19] H. Nakagawa, K. Ohno, and S. Ikeda, "The effect of preoperative subcutaneous fat thickness on surgical site infection risk in patients undergoing colorectal surgery: results of a multisite, prospective cohort study," Ostomy Wound Management, vol. 62, pp. 14-20, 2016.
[20] D. E. Soper and R. C. Bump, "Wound infection after abdominal hysterectomy: effect of the depth of subcutaneous tissue," American Journal of Obstetrics \& Gynecology, vol. 173, no. 2, pp. 465-471, 1995.

[21] J. S. Yudkin, C. D. Stehouwer, and J. J. Emeis, "C-reactive protein in healthy subjects: associations with obesity, insulin resistance, and endothelial dysfunction: a potential role for cytokines originating from adipose tissue?," Arteriosclerosis, Thrombosis, and Vascular Biology, vol. 19, no. 4, pp. 972978, 1999.

[22] M. B. Pepys, "C-reactive protein: a critical update," The Journal of Clinical Investigation, vol. 111, no. 12, pp. 18051812, 2003.

[23] M. Adamina, T. Steffen, I. Tarantino, U. Beutner, B. M. Schmied, and R. Warschkow, "Meta-analysis of the predictive value of C-reactive protein for infectious complications in abdominal surgery," British Journal of Surgery, vol. 102, no. 6, pp. 590-598, 2015.

[24] Z. P. Yang, L. Hong, Q. Wu, K. C. Wu, and D. M. Fan, “Preoperative infliximab use and postoperative complications in Crohn's disease: a systematic review and meta-analysis," International Journal of Surgery, vol. 12, no. 3, pp. 224-230, 2014.

[25] N. Narula and D. Charleton, "Meta-analysis: peri-operative anti-TNF $\alpha$ treatment and post-operative complications in patients with inflammatory bowel disease," Alimentary Pharmacology and Therapeutics, vol. 37, no. 11, pp. 1057-1064, 2013.

[26] G. Rosenfeld and H. Qian, "The risks of post-operative complications following pre-operative infliximab therapy for Crohn's disease in patients undergoing abdominal surgery: a systematic review and meta-analysis," Journal of Crohn's \& Colitis, vol. 7, no. 11, pp. 868-877, 2013.

[27] M. Uchino, H. Ikeuchi, H. Matsuoka et al., "Risk factors for surgical site infection and association with infliximab administration during surgery for Crohn's disease," Diseases of the Colon \& Rectum, vol. 56, no. 10, pp. 1156-1165, 2013.

[28] A. Alves, Y. Panis, Y. Bouhnik, M. Pocard, E. Vicaut, and P. Valleur, "Risk factors for intra-abdominal septic complications after a first ileocecal resection for Crohn's disease: a multivariate analysis in 161 consecutive patients," Diseases of the Colon \& Rectum, vol. 50, no. 3, pp. 331-336, 2007. 


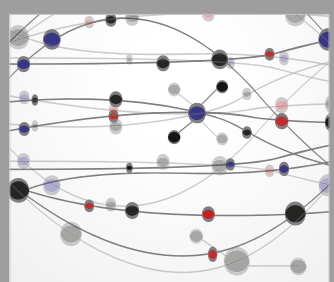

The Scientific World Journal
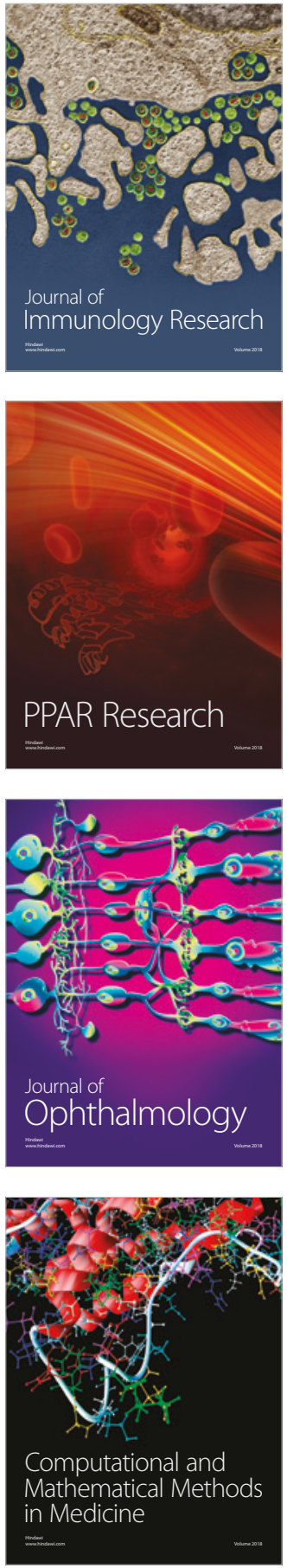

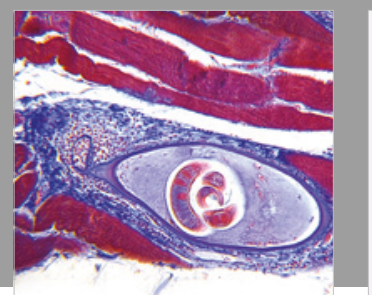

Gastroenterology Research and Practice

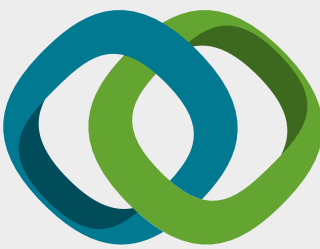

\section{Hindawi}

Submit your manuscripts at

www.hindawi.com
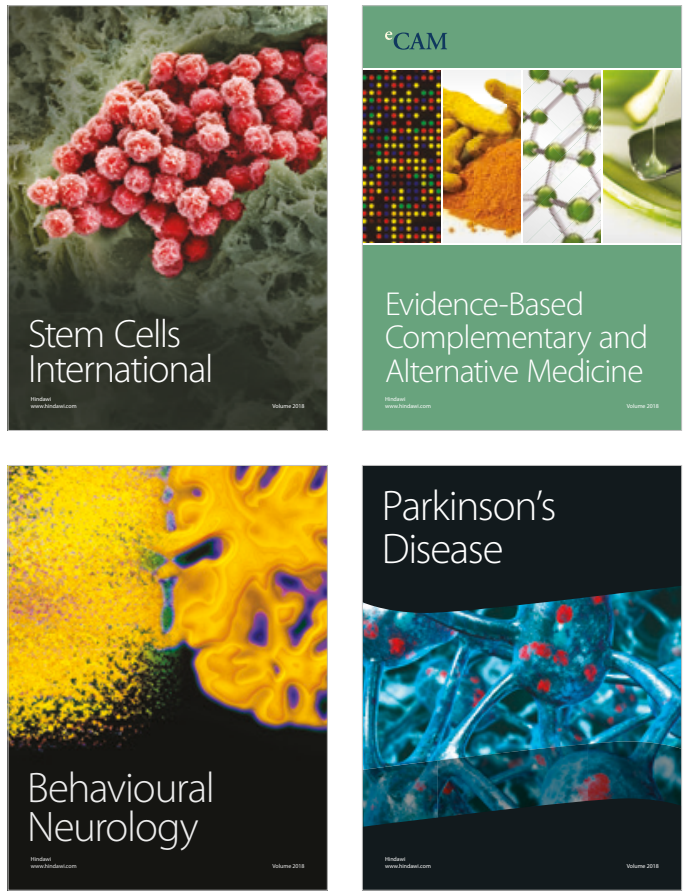

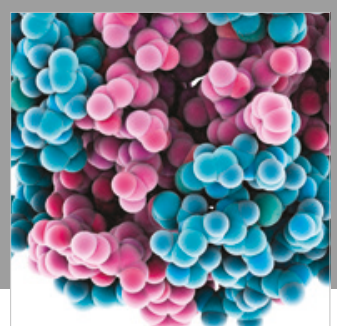

ournal of

Diabetes Research

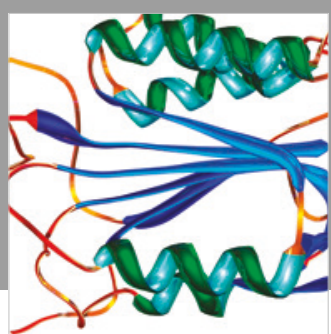

Disease Markers
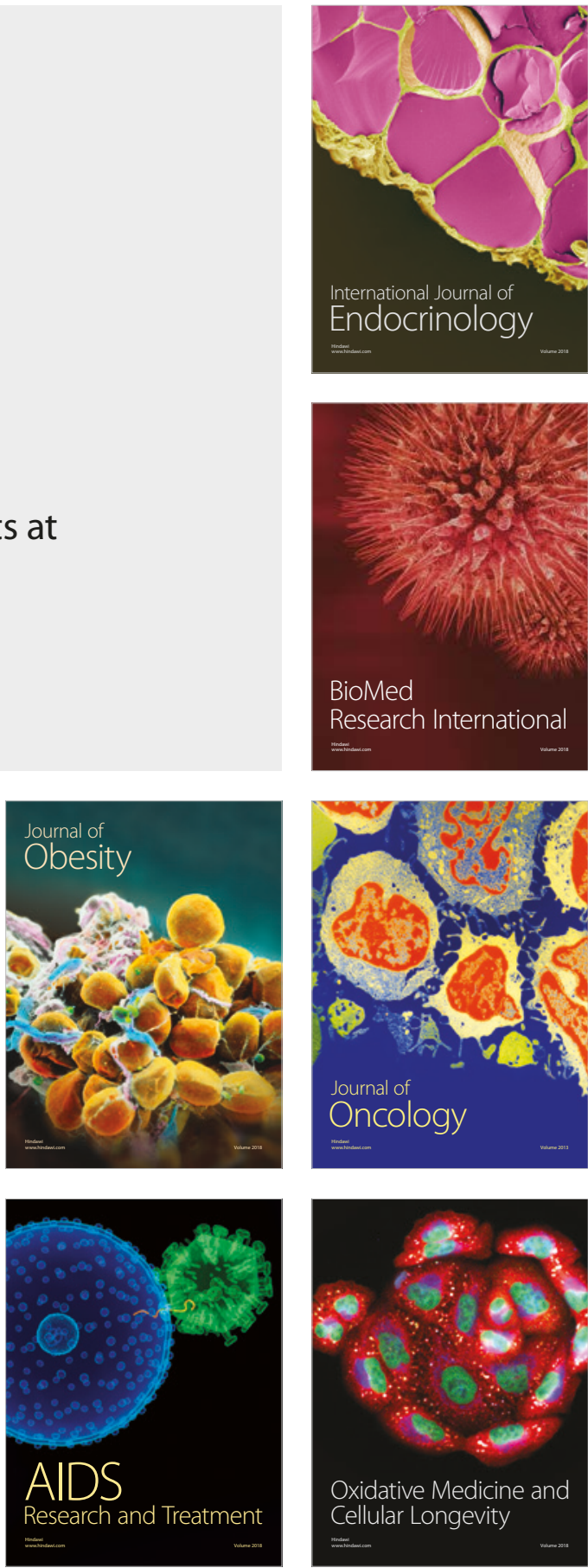